\title{
Lepra Reaction
}

\section{EDITORIALS}

The word "reaction" is used in different senses in leprosy. A delayed local reaction to intradermally injected purified lepromin, if it occurs in a subject who has not had previous contact with leprosy, may be counted as either natural cellular reaction to the lepra bacillus, or the result of previous sensitization by some other mycobacterium. If the subject has had contact with leprosy, or is suffering from early tuberculoid leprosy, the reaction may be enhanced, presumably because he has been sensitized by a previous infection. But if the lepromin test be applied in a lepromatous patient the reaction is negative, and naturally so; because if the millions of bacilli already in the tissues do not cause a reaction then why should the comparatively few bacilli injected do so? And yet this lepromatous patient may, for one of a dozen causes, or for no known cause at all, suddenly begin to react to the bacilli in his tissues and may react most violently. This reactive phase is generally known as "lepra reaction". What causes it? Is it the result of sudden sensitization? If so, it is surely a very different kind of sensitization from that which sensitized the leprosy contact or the early tuberculoid case referred to above. Also a condition very similar to this naturally occurring lepra reaction may be caused equally suddenly by a sufficient oral dose of iodide; and this reaction will pass off as the iodide is eliminated from the body. If this action of iodide could be understood then it might form a key to understanding the nature of lepra reaction. Two things may be asserted about this action of iodide: (I) it is not dependent on the action of iodine, as large quantities of iodine suspended in oil can be injected without causing reaction; (2) iodide does not act directly in the tissues t) cause reaction, as intradermal injections of iodide solution, sufficient to cause a high local concentration, does not cause a local reaction.

In the last few years corticotrophin and cortisone have come into use in the control of lepra reaction. It is possible that iodide acts on the endocrine system to produce effects the opposite of corticotrophin and cortisone? This seems to point to a fruitful line of investigation.

Lepra reaction is often precipitated by accompanying diseases which lower the patient's resistance; but, on the other hand, certain grave weakening conditions, such as enteric and dysentery, nave the effect of suppressing reaction to the extent that nodules and other clinical signs of leprosy rapidly disappear, but appear 
again generally in aggravated form as the patient recovers. This is apparently due to temporary weakening of the tissue cells to the extent that they cannot react to the bacilli in their neighbourhood. 'This phenomenon also requires careful investigation.

The paper by Jopling and Cochrane in this issue gives a clear exposition of the useful role of cortisone and corticotrophin in the control of lepra reaction. In earlier reports of this form of endocrine treatment dramatically effective results were recorded, but the effects were not lasting and reaction returned when administration was stopped. Also excessive doses were at first given. Later workers have shown that equally good but continuing results can be obtained with smaller doses carried on over an indefinite period. This has been confirmed by the authors of the present paper, who also find that sulphone administration does not need to be suspended during endocrine treatment, though the dose may be diminished if reaction is severe. If these findings are confirmed then it should be possible to continue sulphone treatment with less interruption, with more rapid progress, and with much greater comfort to the patient.

One difficulty at present is expense. It may be that the new synthetic steroids mentioned in this paper will be less costly in course of time. We also await further trials of phenylbutazone, favourably reported on by Destombes and Chambon (Lep. Rev., July 1956, p. I36). If these are favourable we should be able to control much more effectively the most distressing of all the complications of leprosy.

\section{International Congress of Leprology}

The date and location of this congress are now finally fixed. It is to be held in New Delhi, India, during the week beginning the 7 th of December, 1958 .

\section{Correction}

On page 139, line 4, of the last issue, "lepra " should read "lepromin". 